\title{
Noise Characteristics of the FORE+OSEM(DB) Reconstruction Method for the MiCES PET Scanner
}

\author{
Kisung Lee, Member, IEEE, Paul E. Kinahan, Senior Member, Robert S. Miyaoka, Member, IEEE, \\ Jeffrey A. Fessler, Senior Member, IEEE, and Tom K. Lewellen, Senior Member, IEEE
}

\begin{abstract}
The FORE+OSEM(DB) image reconstruction method has been proposed for the fully-3D MiCES PET scanner under construction at the University of Washington. It is based on Fourier rebinning followed by 2D OSEM and an incorporated model of detector blurring (DB). As an extension, this paper presents the noise/resolution characteristics of this method. Multiple realizations were simulated to estimate the noise properties of the algorithm. The results are compared with OSEM followed by post reconstruction 3D Gaussian smoothing. The results show that the incorporation of detector blurring (OSEM(DB)) into the system matrix improves resolution compared to OSEM, while also inducing an increased variance at all radial locations. In addition, radially-varying noise characteristics are more apparent with OSEM(DB) than with OSEM.
\end{abstract}

\section{INTRODUCTION}

The Micro Crystal Element Scanner (MiCES) [1] is a small animal PET system dedicated for mouse-imaging that is under development at the University of Washington. We have previously proposed a pragmatic approach to image reconstruction, based on FORE+OSEM with a factorized system matrix approach [2]. The method incorporates detector blurring (DB) PSFs into the system model as a factorized matrix. In this paper, as an extension of our previous image reconstruction study, we investigate noise characteristics [7-9] of the OSEM(DB) method by simulation studies.

\section{MATERIAL AND METHODS}

\section{A. The MiCES scanner and a simplified model}

The full MiCES scanner will consist of 4 rings $(12 \mathrm{~cm}$ inner diameter) of modules, with each ring comprised of 18 detector modules (Fig.1 (a)). The scanner utilizes a total of 72 photo multiplier tubes (PMTs) (1 per module x 4 rings), each coupled to a $22 \times 22$ array of $0.8 \times 0.8 \times 10 \mathrm{~mm}$ discrete mixed lutetium silicate (MLS) crystals. There is a $0.1 \mathrm{~mm}$ inter-crystal gap between adjacent crystals. In modeling the scanner for this study, the target detector modules were simplified as shown in

This work was supported by NIH grants CA-74135, CA-86892, and EB0217.
Fig. 1 (b). In the simplified model, each ring was divided into 396 equally spaced discrete crystals ( 22 crystals x 18 blocks) along the circumference of the ring. In the axial direction, 4 rings were split into 88 crystals ( 22 crystals $\times 4$ blocks). Keeping the same detector ring diameter, the crystal crosssections were $1 \times 1 \mathrm{~mm}^{2}$. No gap was considered between adjacent crystals and the length of the crystals was $10 \mathrm{~mm}$.

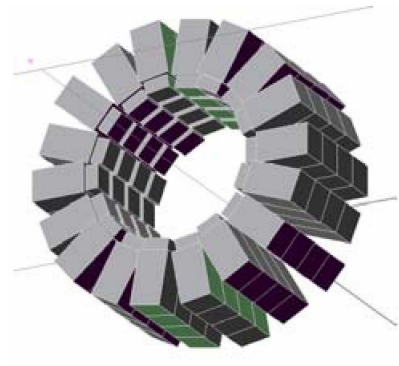

(a)

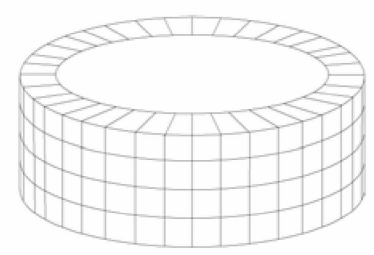

(b)
Fig. 1. (a) Detector module arrangement for the MiCES scanner, (b) simplified model of (a) for the simulations.

\section{B. Number of iterations}

Before investigating the noise characteristics of the OSEM(DB) method, we determined the behavior of OSEM and OSEM(DB) with increasing iteration to determine reasonable stopping points for each method.

We used a test phantom that contained cold and hot spheres with a $6 \mathrm{~mm}$ diameter in a cylindrical background of $25 \mathrm{~mm}$ diameter and $88 \mathrm{~mm}$ length as shown in Fig. 2. The activity ratio of the hot sphere to the background object was 2 to 1 . For the cold sphere, the ratio was 0.5 to 1 .

The analytical simulation tool (ASIM) [3] was used for this study. The phantom objects were numerically forwardprojected into a set of 175 direct 2D sinograms, where each sinogram was 198 by 198 (distance bins by angle bins). The sinogram dimensions were selected to reflect the specifications of MiCES system. The slice thickness and distance bins were 0.5 and $0.3 \mathrm{~mm}$ respectively. The parallel projection planes (i.e., stacks of 2D direct slice projections) were convolved with a 2D positron range PSF. The empirical exponential functions 
by Derenzo were used for implementing the blurring effects of ${ }^{18} \mathrm{~F}$ positron range [4]. Then, those projection planes were blurred again with a spatially-varying detector resolution estimated with the SimSET photon-tracking simulation tool [5]. Poisson noise was then added to each of the sinogram bins. The level of Poisson noise investigated was appropriate for $100 \mathrm{M}$ total counts.

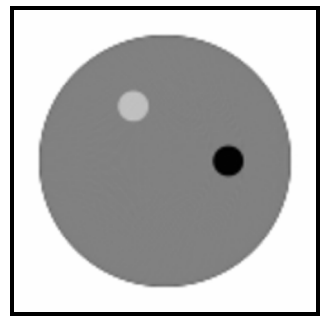

Fig. 2. Background and foreground objects to determine number of iterations

The 2D slices were reconstructed using OSEM and OSEM(DB) with a total of up to 450 iterations (9 subsets x 50 iterations). To model the effect of spatially varying detector blurring (DB) in the FORE+OSEM(DB) algorithm, we have utilized a dynamic linked library for the factorized system matrix in the ASPIRE reconstruction package [6]. The final images had $128 \times 128 \times 175$ voxels with $0.5 \mathrm{~mm}$ axial thickness and $0.47 \mathrm{~mm}$ to a side in a transverse direction.

After each iteration (9 subsets x 1 iteration), ROIs for hot and cold spheres are used to calculate mean values of the spheres. A background ROI was also used with the same size as the spheres for the mean of the background.

\section{Investigation of noise characteristics}

The test phantom for this study consisted of three small spheres (Fig. 4) in a $25 \mathrm{~mm}$ diameter by $88 \mathrm{~mm}$ long cylinder, reflecting the entire field of view (FOV) of the MiCES system. Each sphere was $0.004 \mathrm{cc}$ ( $2 \mathrm{~mm}$ diameter). The sphere-tobackground ratio was set to $4: 1$ and the sphere centers had a 0 , $11,22 \mathrm{~mm}$ offset from the center of the phantom in the transaxial direction.

In the same manner as the section II.B, fifty realizations of the sinogram were simulated by ASIM for the case with the PSF for ${ }^{18} \mathrm{~F}$ positron range, detector blurring, and Poisson noise level (100M total counts). From these images, aspects of noise properties for both OSEM and OSEM(DB) were investigated.

\section{RESULTS}

\section{A. Number of iterations}

The results of the iteration study are shown in Fig. 3. For both hot (Fig. 3(a)) and cold (Fig. 3(b)) spheres, OSEM becomes stable with smaller number of iterations than OSEM(DB). Thus the appropriate iterations for further study were determined to 72 and 90 for OSEM and OSEM(DB) respectively.

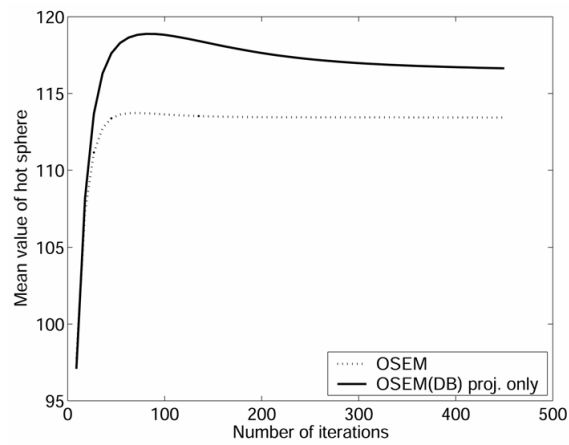

(a)

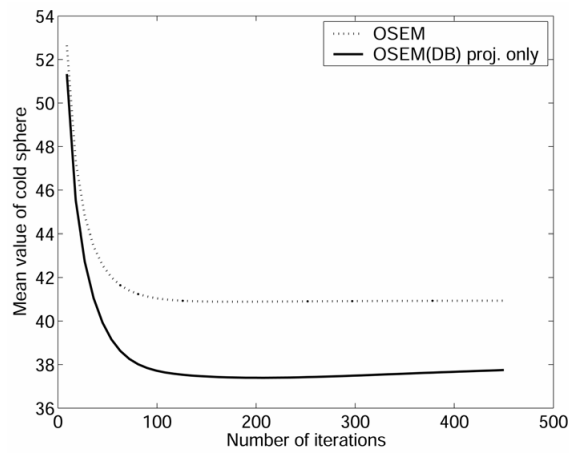

(b)

Fig. 3. Number of iterations vs. mean value of spheres. (a) hot sphere (b) cold sphere shown in Fig. 2.

\section{B. Investigation of noise characteristics}

OSEM reconstruction was performed on the fifty simulated sinogram sets with 9 subsets and 8 iterations while OSEM(DB) was done with the same subsets and 10 iterations.

Mean and variance images are shown in Figs. 4 and 5 respectively. No post-filtering were applied to generate mean and variance images. OSEM(DB) resulted in increased variance in the FOV boundary region and the value increased with radial distance, while OSEM images were not sensitive to the radial position. This effect is likely caused by the radiallyvarying detector PSFs incorporated in OSEM(DB) method (i.e., the PSF at the center is much sharper than at the FOV boundary).

Fig. 6 shows profiles of noiseless, mean, and variance images along the transverse axis with FBP, OSEM, and OSEM(DB) reconstruction methods. OSEM(DB) improves contrast of recovered hot spheres in all radial positions while FBP and OSEM produce similar results. For variance, OSEM(DB) increases the noise with the radial distance while OSEM is lower compared to FBP. In addition OSEM(DB) induces small ripple effects around object boundaries, (Fig. 6 (a)). 

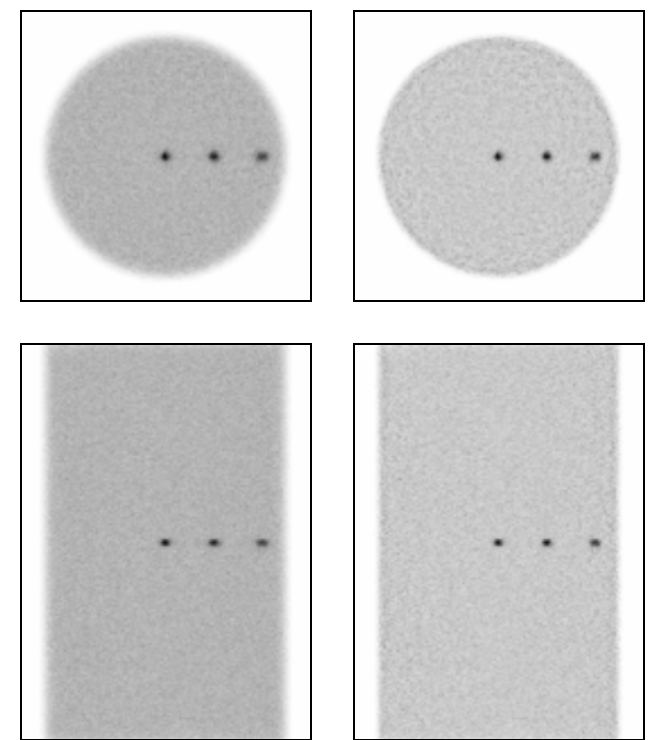

Fig. 4. Mean image volumes from 50 realizations by OSEM(left) and OSEM(DB)(right). Upper images are transverse slices and lower images are coronal sections. The images are scaled from 0 to maximum resulting in a different gray scale for OSEM and OSEM(DB).
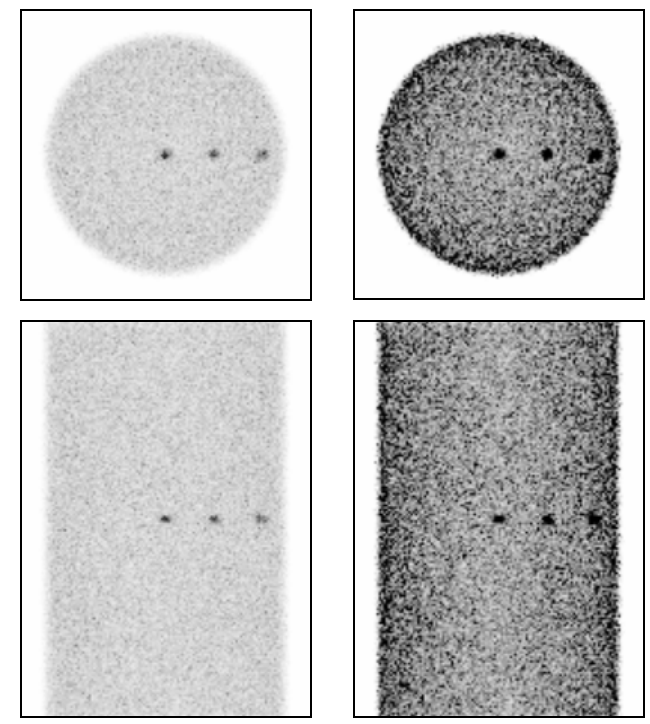

Fig. 5. Variance image volumes from 50 realizations by OSEM(left) and OSEM(DB)(right).

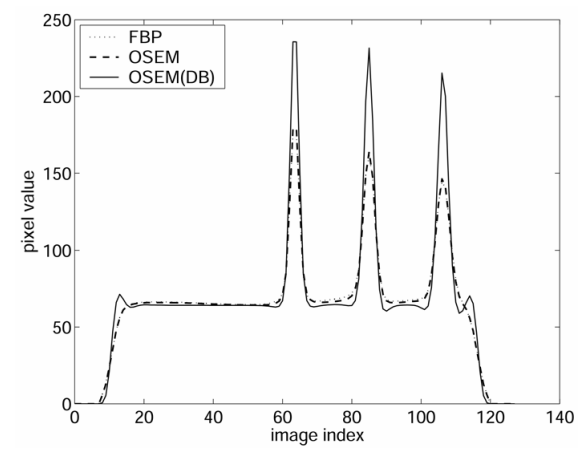

(a)

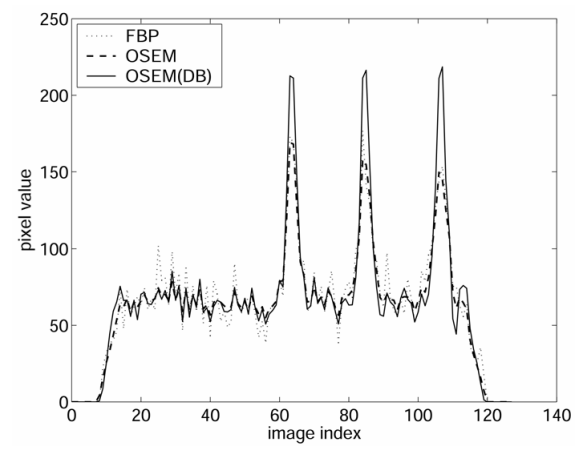

(b)

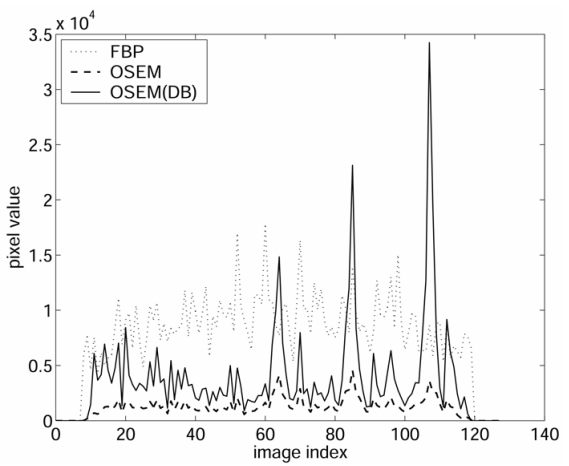

(c)

Fig. 6. Transverse profiles of (a) noiseless, (b) mean, and (c) variance images.

Tables I and II list quantitative measurements of mean and variance in target spheres and background ROIs at the FOV center and at 11 and $22 \mathrm{~mm}$ off-center. The three hot spheres were selected as foreground ROIs. Spheres of the same size but shifted axially $22 \mathrm{~mm}$ from the axial center were chosen as background ROIs. No post filtering was applied to both OSEM and OSEM(DB) reconstruction while FBP utilized a ramp filter. The OSEM(DB) results in Table I and II show improved contrast over FBP and OSEM. However, OSEM(DB) induces a increased noise (variance) in foreground and background ROI spheres at all radial locations than the two other methods. In addition, radially-varying noise is more apparent in OSEM(DB) for both target and background ROIs. 
Table III shows the full width half maximum (FWHM) values of the three hot spheres reconstructed by FBP, OSEM, and OSEM(DB). The results shows that OSEM(DB) improves resolution at all radial distances and enhances the resolution more significantly as radial distance increases.

TABLE I

ROI MEAN FROM FIFTY REALIZATIONS

\begin{tabular}{|c|c|c|c|c|}
\hline & & center & $\begin{array}{c}12 \mathrm{~mm} \\
\text { offset }\end{array}$ & $\begin{array}{c}24 \mathrm{~mm} \\
\text { offset }\end{array}$ \\
\hline \multirow{2}{*}{ FBP } & hot sphere & 153.5 & 152.7 & 137.0 \\
\hline & $\begin{array}{c}\text { background } \\
\text { ROI }\end{array}$ & 63.6 & 64.5 & 68.6 \\
\hline \multirow{2}{*}{ OSEM } & hot sphere & 149.5 & 151.0 & 135.2 \\
\hline & $\begin{array}{c}\text { background } \\
\text { ROI }\end{array}$ & 65.0 & 64.8 & 67.0 \\
\hline \multirow{2}{*}{ OSEM(DB) } & hot sphere & 183.4 & 195.9 & 178.5 \\
\hline & $\begin{array}{c}\text { background } \\
\text { ROI }\end{array}$ & 63.6 & 64.1 & 65.8 \\
\hline
\end{tabular}

TABLE II

ROI VARIANCE FROM FIFTY REALIZATIONS

\begin{tabular}{c|c|ccc}
\hline \multicolumn{2}{c|}{} & center & $\begin{array}{c}12 \mathrm{~mm} \\
\text { offset }\end{array}$ & $\begin{array}{c}24 \mathrm{~mm} \\
\text { offset }\end{array}$ \\
\hline \multirow{2}{*}{ FBP } & $\begin{array}{c}\text { hot sphere } \\
\text { background } \\
\text { ROI }\end{array}$ & 114.2 & 201.2 & 161.8 \\
\hline \multirow{2}{*}{ OSEM(DB) } & $\begin{array}{c}\text { hot sphere } \\
\text { background } \\
\text { ROI }\end{array}$ & 78.9 & 94.8 & 106.8 \\
& $\begin{array}{c}\text { hot sphere } \\
\text { background } \\
\text { ROI }\end{array}$ & 218.5 & 338.0 & 57.7 \\
\hline
\end{tabular}

TABLE III

ROI FWHM OF MEAN IMAGE (MM)

\begin{tabular}{l|ccc}
\hline & center & $\begin{array}{c}11 \mathrm{~mm} \\
\text { offset }\end{array}$ & $\begin{array}{c}22 \mathrm{~mm} \\
\text { offset }\end{array}$ \\
\hline FBP & 1.88 & 2.25 & 2.48 \\
OSEM & 1.73 & 2.06 & 2.34 \\
OSEM(DB) & 1.64 & 1.69 & 1.73 \\
\hline
\end{tabular}

Fig. 7 shows noise versus bias plots for the target spheres at the FOV center and $11 \mathrm{~mm}$ and $22 \mathrm{~mm}$ offset positions. For FBP, Hanning filters with 1.0, 0.8, 0.6 cutoffs were used respectively. OSEM and OSEM(DB) were post-processed by 3D Gaussian filter with 0, 0.47, 0.94, 1.41, 1.88, $2.34 \mathrm{~mm}$ FWHM respectively. For the spheres at all three radial distances, the bias is decreased at the expense of increased noise.

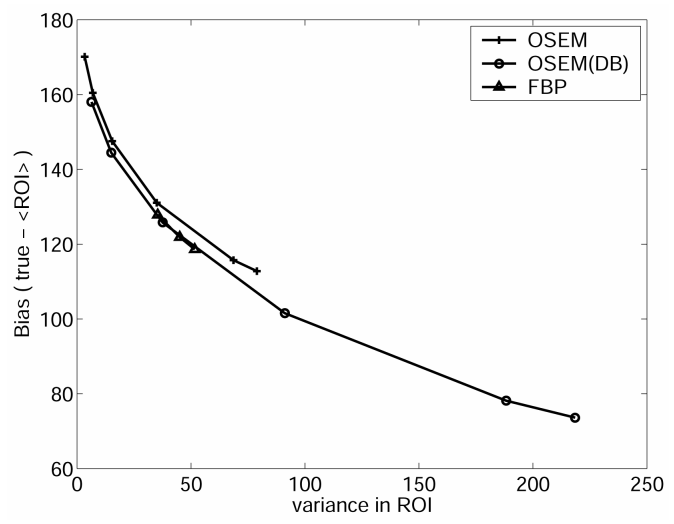

(a)

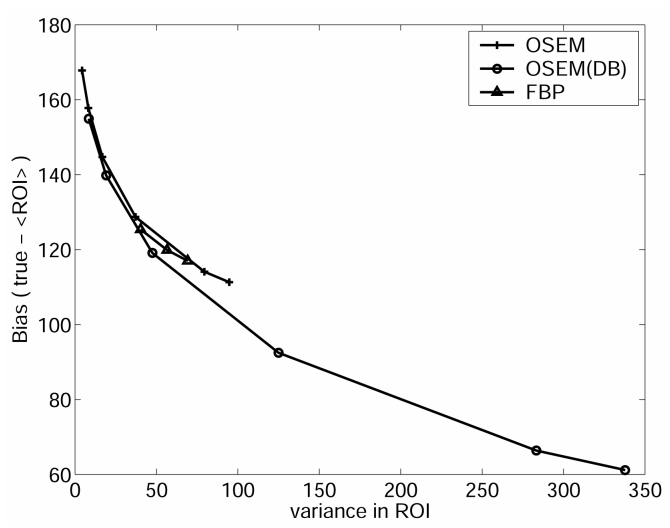

(b)

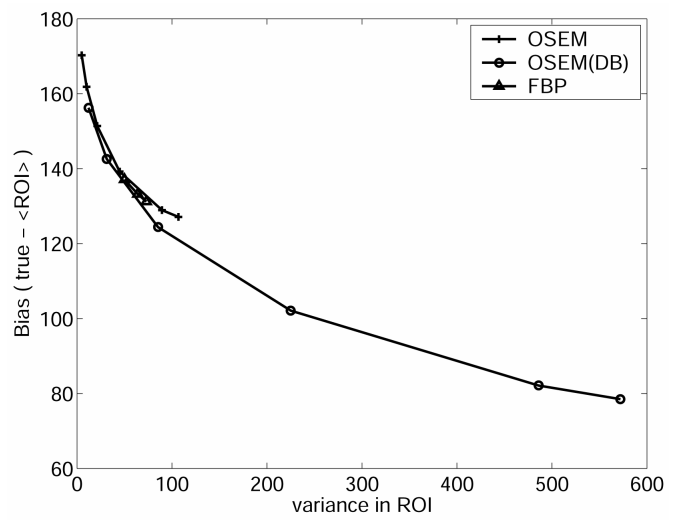

(c)

Fig. 7. Noise vs. bias of foreground ROIs. (a) FOV center, (b) $11 \mathrm{~mm}$ offset, and (c) $22 \mathrm{~mm}$ offset

Fig. 8 shows the noise versus root mean square error (RMSE) plots for the foreground spheres at FOV center, 11 $\mathrm{mm}$, and $22 \mathrm{~mm}$ offset positions. 


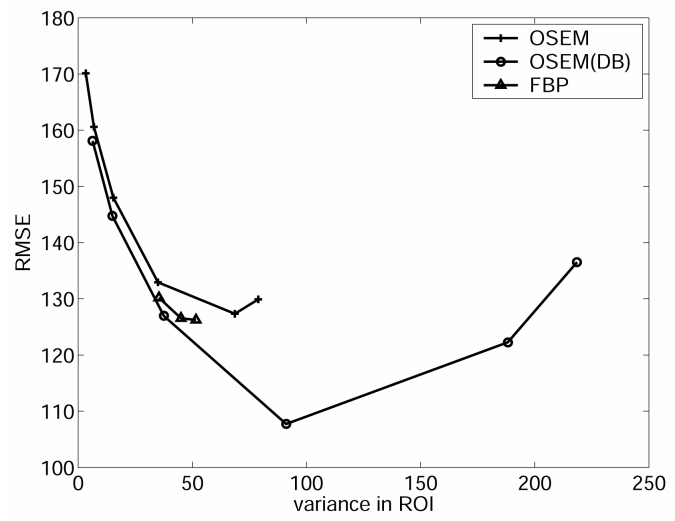

(a)

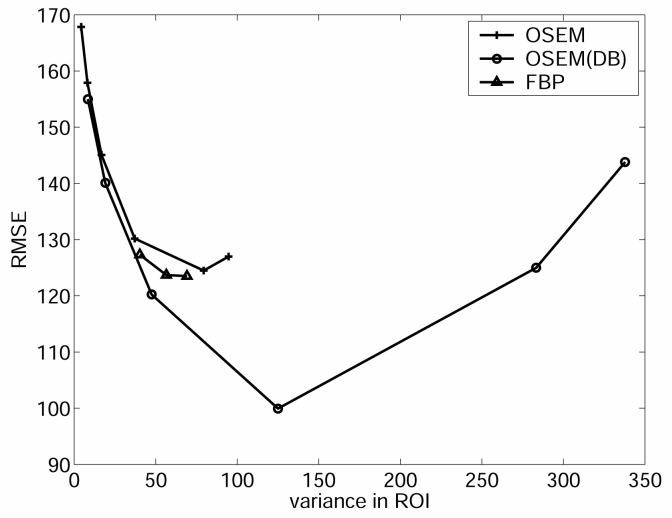

(b)

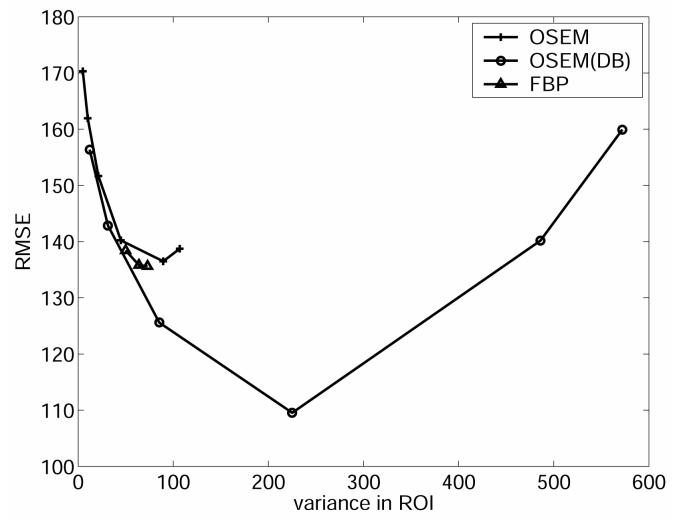

(c)

Fig. 8. Noise vs. RMSE of foreground ROIs. (a) FOV center, (b) 11 $\mathrm{mm}$ offset, and (c) $22 \mathrm{~mm}$ offset

\section{DISCUSSION}

In this study we investigated the noise characteristics of OSEM(DB) reconstruction method for the MiCES mouse imaging PET scanner.
The results show that the incorporation of detector blurring (OSEM(DB)) into the system matrix improves resolution compared to OSEM, while also inducing an increased variance at all radial locations. In addition, radially-varying noise characteristics are more apparent with OSEM(DB) than for OSEM for both target and background ROIs.

The focus of this study is to investigate the noise characteristics by involving the detector blurring effect in the system matrix. Thus we used only direct planes for the sinograms rather than using FORE.

The current OSEM(DB) reconstruction applied detector blurring effect into the system matrix only in forward projection. In addition, further investigation of noise vs. bias/RMSE will be performed with higher variance values using OSEM.

\section{REFERENCES}

[1] T. K. Lewellen, M. Janes, R. S. Miyaoka, S. B. Gillespie, B. Park, K. S. Lee, P. Kinahan, "System integration of the MiCES small animal PET scanner," IEEE Nuclear Science Symposium and Medical Imaging Conference, , in press, Rome, Italy, Oct. 2004.

[2] K. Lee, P. E. Kinahan, J. A. Fessler, R. S. Miyaoka, and T. K. Lewellen, "Pragmatic Image Reconstruction for the MiCES Fully-3D Mouse Imaging PET Scanner," IEEE Nuclear Science Symposium and Medical Imaging Conference, conference record in CD, Portland, OR, Oct 19-25, 2003.

[3] C. Comtat, P. E. Kinahan, M. Defrise, C. Michel, C. Lartizien, and D. W. Townsend, "Simulating whole-body PET scanning with rapid analytical methods," in IEEE Nuclear Science Symposium and Medical Imaging Conference, vol.3, pp. 1260-1264, 1999.

[4] S. E. Derenzo, "Precision measurement of annihilation point spread distributions for medically important positron emitters," in Proc. of the $5^{\text {th }}$ Int. Conf. Positron Annihilation, pp. 819-823, Japan, 1979.

[5] http://depts.washington.edu/ simset/html/simset main.html, SimSET homepage

[6] J. A. Fessler, Dynamic Libraries for ASPIRE Penalty Functions and System Models Communications \& Signal Processing Laboratory, University of Michigan, 2003.

[7] V.V. Selivanov, Y. Picard, J. Cadorette, S. Rodrigue, and R. Lecomte, "Detector Response Models for Statistical Iterative Image Reconstruction in High Resolution PET, "IEEE Transactions on Nuclear Science, Vol. 47, No. 3, June 2000

[8] J. Qi and R. M. Leahy, "Resolution and Noise Properties of MAP Reconstruction for Fully 3-D PET," IEEE Transactions on Medical Imaging, Vol. 19, No. 5, May 2000.

[9] T. Frese, N. C. Rouze, C. A. Bouman, K. Sauer, G. D. Hutchins, "Quantitative Comparison of FBP, EM, and Bayesian Reconstruction Algorithms for the IndyPET Scanner", IEEE Transactions on Medical Imaging, Vol. 22, No. 2, February 2003. 